\title{
Diffuse leptomeningeal melanocytosis
}

INSERM

\section{Source}

INSERM. (1999). Orphanet: an online rare disease and orphan drug data base. Diffuse leptomeningeal melanocytosis. ORPHA:252031

Diffuse leptomening eal melanocytosis is a rare tumor of meninges arising from leptomeningeal melanocytes, characterized by diffuse infiltration of the leptomeninges (pia mater and arachnoidea) anywhere in the central nervous system. Clinical features may include stillbirth, intracranial hypertension and hydrocephalus, seizure, ataxia, syring omyelia, cranial nerve palsy, intracranial haemorrhage, sphincter dysfunction and neuropsychiatric symptoms. Transformation into malignant melanoma of the central nervous system was reported. It may be associated with congenital nevi, as a part of neurocutaneous melanosis. 